\title{
Bottleneck Detection and Reduction Using Simulation Modeling to Reduce Overcrowding of Hospital Emergency Department
}

\author{
Qingjin Peng, ${ }^{1, *}$ Jie Yang ${ }^{1}$, Trevor Strome², Erin Weldon², Alecs Chochinov² \\ 1. Department of Mechanical Engineering, University of Manitoba, Winnipeg, MB, Canada \\ 2. Winnipeg Regional Health Authority Emergency Program and Department of Emergency Medicine, \\ University of Manitoba, MB, Canada \\ Email: Qingjin.Peng@umanitoba.ca (Corresponding author)
}

Received: 30 April 2020; Accepted: 8 June 2020; Available online: 25 July 2020

\begin{abstract}
Overcrowding is a common problem in hospital emergency departments (EDs) where the ED service cannot meet care demands within reasonable time frames. This paper introduces a quantitative approach using computer simulation modeling for hospital decision makers to explore trade-offs between efficiency, workload and capacity of EDs. A computer simulation model is built based on the ED of a local hospital to improvement efficiency of the ED patient flow. Bottlenecks of the emergency care process are detected using the built model. The ED performance is examined by applying alternative strategies to reduce patient waiting time and length of stay. The proposed method can be applied to improve the operation efficiency of healthcare systems in the current pandemic, COVID -19.
\end{abstract}

Keywords: Bottleneck detection; Simulation modeling; Emergency department; Overcrowding.

\section{Introduction}

Hospital emergency departments (EDs) provide the emergency medical treatment for patients. In Canada, 10\% of the patients are admitted to the hospital through EDs according to a report of the Canadian Institute for Health Information [1]. Most patients spend approximately 7.5 hours in EDs, but for $10 \%$ patients who need to be admitted, their stays in EDs can be much longer.

A common term ED overcrowding is often used when studying waiting time in EDs, which refers to a situation where the ED service cannot meet care demands within reasonable time frames [2]. Overcrowding in EDs has been a common complaint in Emergency Medicine in Canada for more than 20 years [3]. Considering its adverse effects on patient satisfaction and safety, improving ED operations to alleviate ED overcrowding becomes crucial and urgent [4-7].

ED overcrowding is a complex public health concern, its leading factors penetrate in almost every level of the health care system [3]. But in overall, the mismatch between the supply and demand in health care systems devotes the major contribution to this issue [2]. In the past decades, researchers have made great efforts in attempt to define a single universal standard to quantify the ED crowdedness, such calculators as NEDOCS (National Emergency Department Overcrowding Study) and EDWIN (Emergency Department Work Index). But more studies are needed to test whether these scales can provide a good reflection of the overcrowding at other EDs before they are determined to be universal standards [8].

Previous studies indicate that one of the main factors causing ED overcrowding is the process inefficiency within the ED. To improve the patient flow within the ED, multiple strategies have been proposed for potential solutions, such as ED floor plan modifications, changes in workflows, and resources adjustments. But these solutions cannot be directly applied for an ED as each ED has its specific problem related to patients and resources [2].

This research is undertaken at a local hospital in Winnipeg, Canada. The hospital management considers implementing some changes to reduce patient waiting time and throughput time in their emergency care process in order to improve the overall service delivery and system throughput.

Due to complexity of the ED system, decisions need to be much more evidence-based and made in near-realtime conditions. Simulation modeling is an effective tool for analyzing and identifying the variability in EDs. Simulation modeling, as one of the most commonly used operation research techniques, has been widely applied in identifying various issues of EDs to improve ED performance in forecasting, capacity planning, resource allocation, staffing and scheduling, measuring metrics, geographic optimization, and supply chain management [9]. By developing a virtual model of real ED systems, the process performance can be investigated. Various 
strategies of the ED operation can be evaluated through model experiments for a better operating policy. Without disturbing the actual system, the simulation model is proved an efficient decision-making tool.

This paper introduces our research using simulation modeling to examine the ED in various conditions. Using the computer simulation, it is proposed to achieve success of modeling the emergency care process and determining the impact of key resources on ED performance such as the patient waiting time and length of stay. Therefore, the objective of this research is to identify and reduce bottlenecks of the ED patient flow using a validated computer model. The simulation model is used as a testbed to examine feasible alternatives to reduce bottlenecks for improvement of the ED performance.

\section{Related research}

\subsection{ED overcrowding}

Although there is a lot of attentions to ED overcrowding in the public, there is not a national standard definition in Canada to measure it. It is true also in many other countries [3, 10-12]. Boyle et al. discussed an international Delphi-based standard that was composed of eight-point measures of ED crowding, called International Crowding Measure in Emergency Department (ICMED) [13]. Higginson grouped the eight-point measures into three categories with specified operational definitions of input, throughput, and output [10].

As noted by many researchers [3, 14], ED overcrowding is a complex and multi-dimensional health service system problem which cannot be solved by examination of the ED in isolation [15, 16]. To identify the ED overcrowding problem, several publications have recommended to examine ED overcrowding in the context of the entire health service delivery system which could be conceptualized using the input-throughput-output model $[3,10]$. The model allows most factors related to overcrowding to be measured.

Although there is not a universal measure of the ED crowdedness, a reliable alternative seems well accepted by the public, which is to measure the ED crowdedness by monitoring outcomes. Some of the commonly studied outcome measures are patient wait time, patient length of stay (LOS), patient throughput, left without being seen (LWBS), bed occupancy, ambulance diversion rate, and resource utilization. A Canadian national survey demonstrated that these outcome measures could be a good projection of the ED crowdedness because their impact on ED crowding was obvious [17].

\subsection{Simulation modeling for improving ED patient flows}

Simulation modeling is one of the most widely used operation research techniques. Its contributions to the ED patient flow have been remarkably extensive. There is a great volume of in-depth research on the ED patient flow using simulation to understand causes of ED overcrowding and to test various interventions to alleviate effects of overcrowding. Simulation shows the great impact on ED processes at care-team, organizational and environmental levels $[18,19]$.

Different research has been conducted to examine patient flows in ED processes for improvement. Most research in the ED simulation falls in one of these four motivations: costs and competition, efficiency, reengineering, and quality of the service [20, 21]. A primary goal of most simulation research is to identify bottlenecks, detect areas of improvement, and test various "what if" strategies. Simulation is also used in addressing common ED issues, generally with respect to forecasting, capacity planning, resource allocation, staffing and scheduling, measuring performance metrics, geographic optimization, and supply chain management $[22,23]$.

Since the computer simulation provides a virtual model of a real or proposed system to examine its reaction to various conditions, it is favored over analytical solutions when studying complex dynamic systems such as an ED $[24,25]$. It can test various "what if" scenarios within a minimum amount of time, which has made simulation a widespread tool $[26,27]$. Comparing with other methods applied in the ED improvement, simulation is much more flexible and versatile as it allows free assumptions of input data (i.e. the arrival pattern and service time). The system structure can be of any complexity and the custom action logic can be built in to mimic the real system behavior in a great level of details [22].

However, the simulation application in EDs has its limitations. It is the ED specific, and not generalizable [18, 28]. Substantial diversity exists among different EDs in terms of the objective, scope, level of details and calibrations. Rather than a generically available tool, the simulation is more of a case-by-case approach [28]. Therefore, a detail investigation has to be conducted for a specific ED operation and its resources in order to using the simulation as an analytic tool for the ED patient flow improvement.

\section{ED patient flow}

The emergency department (ED) investigated in this research is a 24-hour/7-day emergency care facility with approximately 42,000 patient visits annually, which is about 120 patients per day. There are a total of 40 spots 
(beds/seats) for patient care divided into six distinct care areas: Resuscitation Room, Stretcher Bay, ERooms, Minor Treatment Area, Reassessment Unit, and Psych Rooms.

A visit to the ED usually involves a various series of decisions, activities, and interactions with ED and hospital staff. Patient flows can vary from patient to patient based on the acuity level and diagnosis, and thus it is impossible to classify all ED patient flows exactly. However, a general ED patient flow can be determined, which involves most common decisions, activities, and interactions that a patient will experience in the ED. Based on the information provided by the ED staff, a general patient flow chart is constructed as shown in Figure 1. A typical visit to the ED usually involves pre-bed service, on-bed service and departing the ED.

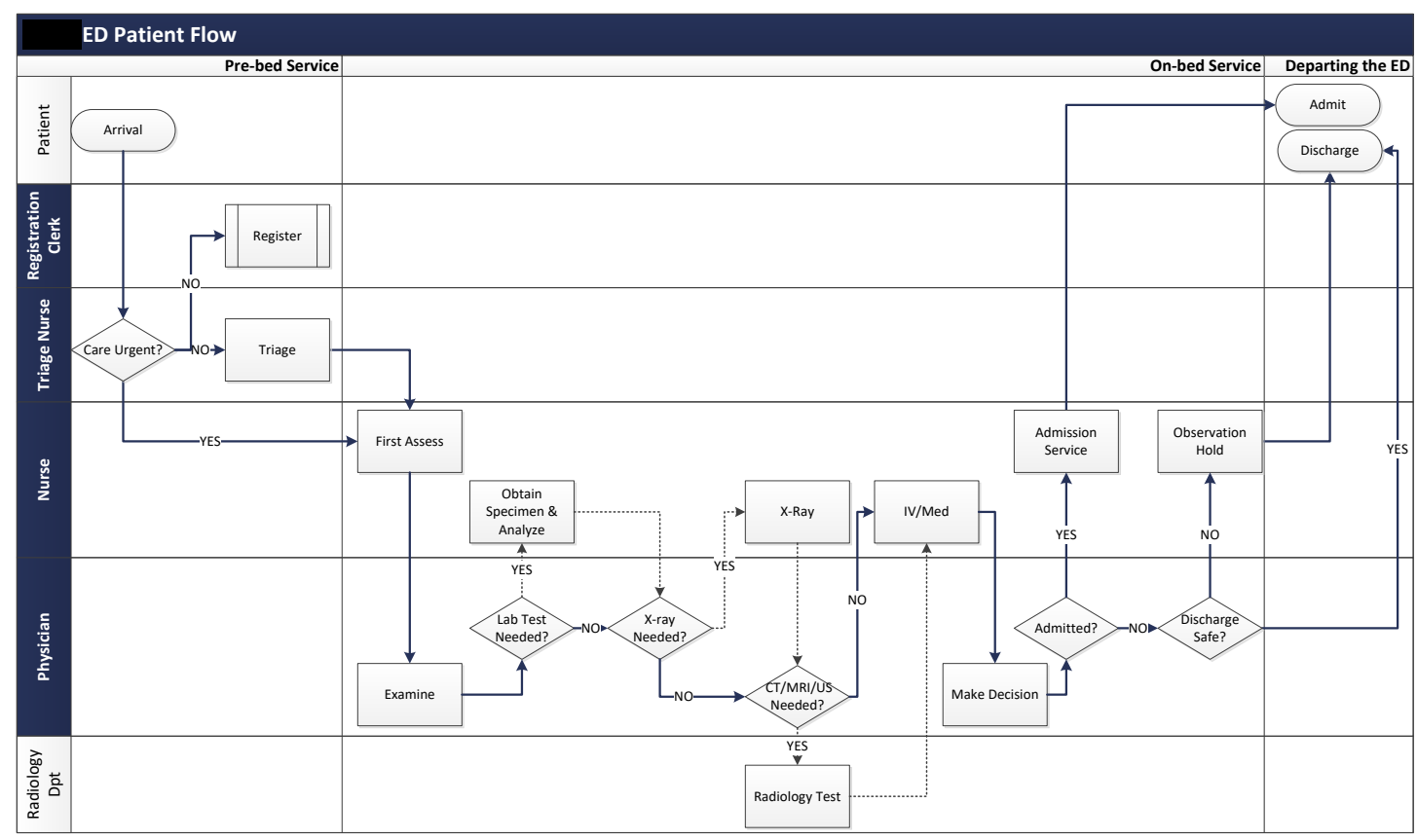

Figure 1. A general patient flow in the ED

\section{ED simulation modeling}

Based on the ED patient flow, input components of the simulation model include factors that contribute to the demand for ED services (i.e. sources of patients seeking the ED care). The throughput component looks internally at the ED operation, specifically in associated with the ED efficiency, workload, and capacity. Output components define bottlenecks that cause delay in patient disposition such as patient boarding in the ED, i.e. a situation where a patient supposed to be admitted hospital remains in the ED due to certain factors.

A discrete-event simulation (DES) model is built to understand the behavior and performance of the ED operation. It requires a validated model to simulate the ED patient flow, conduct the bottleneck analysis to identify factors that prevent an efficient ED, and test various ED operating alternatives for improvements. It is important to understand that the model is developed using a number of assumptions to simplify the modeling effort by eliminating any insignificant parameters and processes. The most significant assumptions used in constructing the model are as follows.

1) All patients remain at their critical level throughout their stay in the ED. The critical level is assigned during triage for patients after entering the ED with emergent conditions.

2) The two psych rooms for mental health patients are not considered in the model, because there are no other types of patients competing for the psych rooms. Patients searching for service in the psych rooms can be regarded as a small isolated event in the ED. Compared to a big majority of other types of patients, mental health patients represent a very small percentage of all patients seen in the ED, and their impact on the total resource utilization and treatment time is very limited and can be ignored.

3) All patients undergo triage and registration on arrival when the resource is available. Emergent patients who bypass triage and registration on arrival have a very tiny proportion $(0.26 \%)$ according to the historical data, and ignoring this would not cause any pronounced effects on the model for the project purpose.

A DES simulation modeling process consists of following steps: the problem formulation, setting objectives, conceptual modeling, data collection, model design and building, model verification and validation, model analysis and solution documentation. 
The first step of model building is to fully understand the ED flow. Based on this understanding, the problem statement is formulated to describe the problem that needs to be solved and defined as an overall goal of the research with specifics of constraints. Following of this, a conceptual model is developed to abstract essential features of the ED system with consideration of the formulated problem and objectives of the simulation research. The conceptual model is then translated into a data-driven computer programmed simulation model in a sequence of ED operations.

In the DES model, the layout, structure, logic, data and statistics of the ED system are built. In this research, simulation software Witness is used to build the model of the ED system. In the ED model, major elements include patients, ED staff, service operations and patient flows.

Historical data of one year ED operation were collected from the ED information system provided by the regional health authority with a total of 39,525 anonymous patient records. Each patient record is described in time, location and status stamps in an excel sheet. In fact, not all collected data were used to build the model. Instead they were divided into two sets. The first set consists of data from the first six months of the total collected data that were used to fit distributions as input data of the model in the model construction, the second data set contains the remaining six months of the data that were used to validate the model.

As the goal of this research is to reduce patient waiting time to see a doctor (WTBS) and their length of stay in the ED (LOS), these two measures need to be robustly validated for modelling intent. To generate more credibility of the model, the time spending for a patient from start of triage until the end of active treatment on an assigned room (i.e. WTBS + TIP) is also strictly validated as well as patient daily throughput, total patient arrivals to ED and total patients released from ED. Figure 2 illustrates the patient journey from arrival to exit with motoring tags (i.e. WTBS, TIP, LOS etc.). ED activities that direct add value to patients are here named as value added activities.

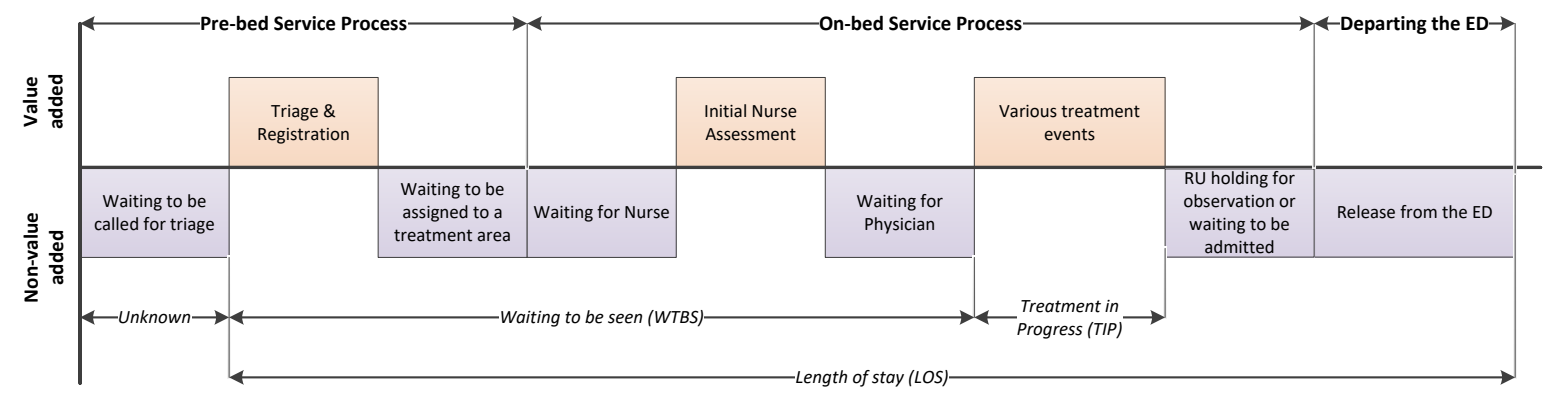

Figure 2. Value stream mapping of the ED activities

The model undergoes the verification and validation during the entire model building process. Model verification is the first step of the quality control check to see if the simulation model is built and performed on the indented logical design. A successful verification reflects what the modeler has initially designed. Model validation is the second step of the quality control check before the model can be used to test various "what if" scenarios and predict future behavior. It is a process of checking the accuracy of the built model to determine if the model is a good representation of the real-world system. Here it should be realized that the model is only validated based on the project purpose, it can never become a 100\% representation of the real ED system. Once the built model is validated for the project purpose, it is ready to be used as a decision making tool to analyze effects of different ED operating scenarios on the ED performance. Figure 3 is a screenshot of the ED model built using the Witness simulation system.

\section{Bottleneck detection}

Bottlenecks have to be identified in order to reduce patient waiting time to see a doctor and their length of stay in the ED. A method of evaluating critical indicators for each process is applied to detect bottlenecks in the existing patient flow [29]. A critical indicator for the ith service station is used to look at the difference of individual rates in the process such as the utilization, starvation, blocking, waiting for operator with respect to the whole system average of this rate as follows.

$$
K R_{i}=\left(\frac{\sum_{i=1}^{n} B_{i}}{n}-B_{i}\right)+\left(I_{i}-\frac{\sum_{i=1}^{n} I_{i}}{n}\right)+\left(B L_{i}-\frac{\sum_{i=1}^{n} B L_{i}}{n}\right)+\left(L_{i}-\frac{\sum_{i=1}^{n} L_{i}}{n}\right)
$$

where $K R_{i}$ is a criticality indicator for the $i$ th service station, $B_{i}$ is the average utilization rate for the $i$ th station, $I_{i}$ is the average starvation rate for the ith station, $B L_{i}$ is the average blocking rate for the ith station, $L_{i}$ is the average waiting rate for operator for the ith station. The critical indicator gives a quantitative identification of the severity 
of each station. The service station with the minimal value of $K R_{i}$ denotes a bottleneck station that may need capacity building, whereas the station with the maximal value of $K R_{i}$ denotes a station with a better utilization.

Critical indicators are found using this method for all service processes in the ED. Graphical results of the bottleneck analysis are shown in Figure 4. It shows that critical values for stations of triage and reassessment unit are extremely low, which implies that the top priority should be given to these two processes in order to have a smoother patient flow. Improvement should also be searched for MTA and ERms as their critical values are also relatively low.

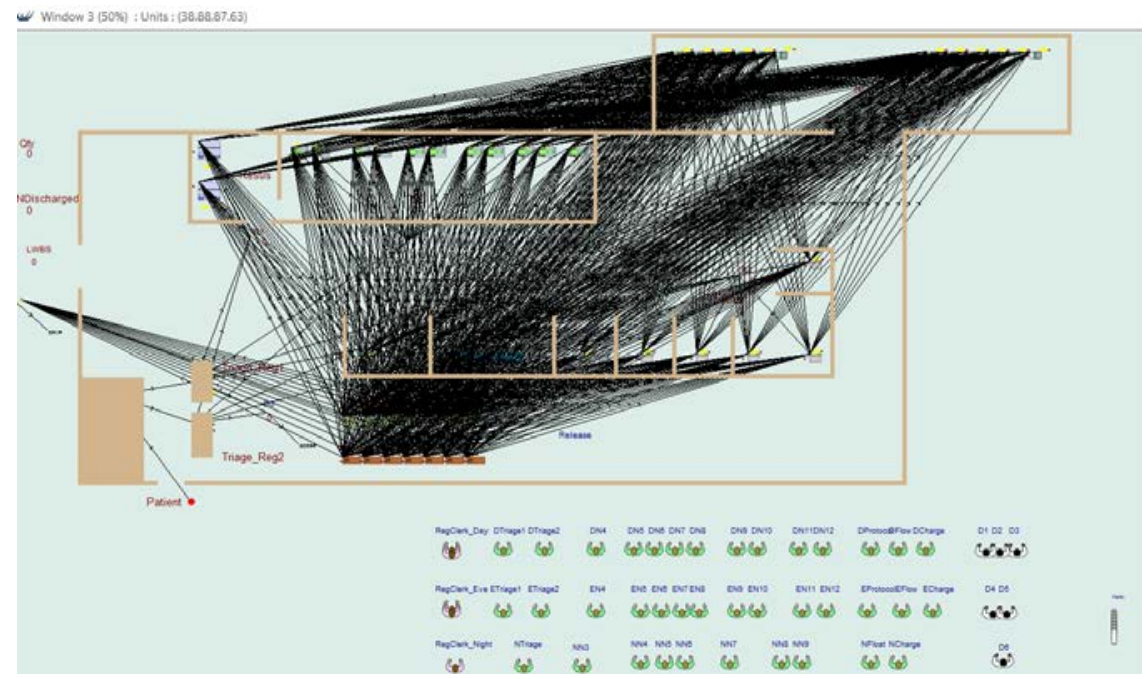

Figure 3. ED simulation model

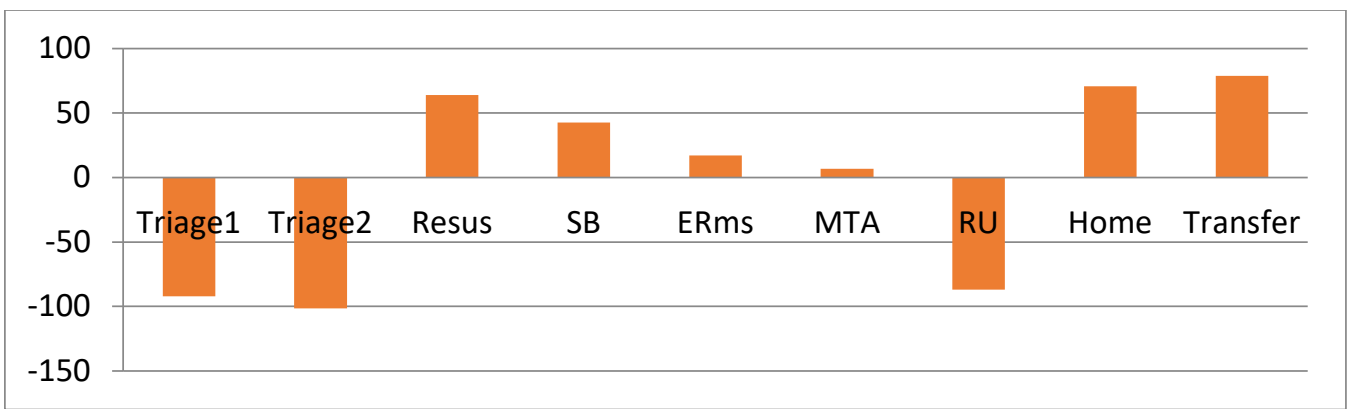

Figure 4. ED bottleneck analysis

Using the same method, critical values are analyzed for all ED physicians and nurses to identify possible resources that cause blockage in the flow. Figure 5 shows that physician \#5, \#6, \#2 and \#3 are potential limiting factors that delay the patient flow. Figures 6 and 7 are graphs that display results of the bottleneck analysis on nurses. On day and evening shifts, nurses share a same room assignment rule whereas nurses on night shift have a different one. Figures 6 and 7 indicate that triage nurse and nurses looking after patients in ERms and MTA could possibly lead to the blockage in the flow as well.

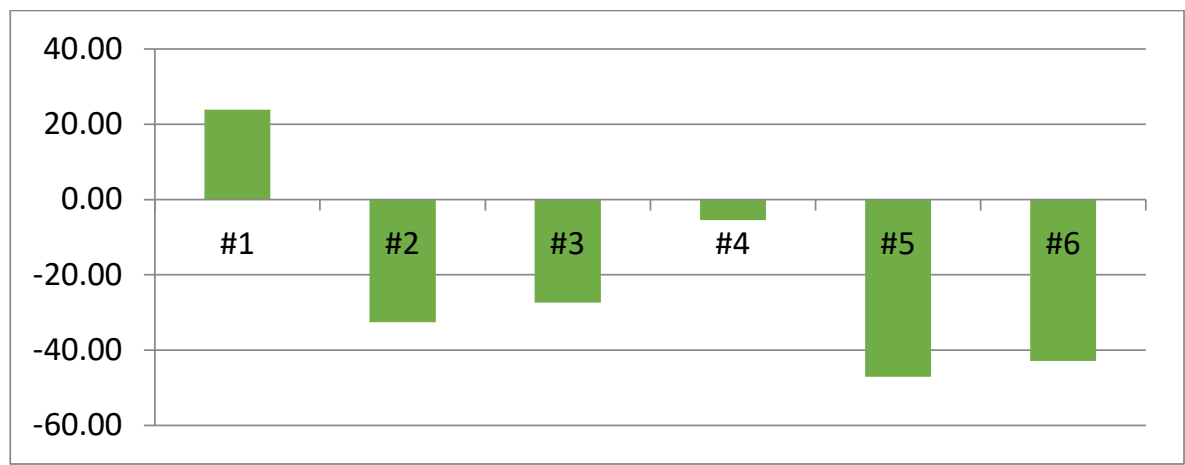

Figure 5. Bottleneck analysis on ED physicians 


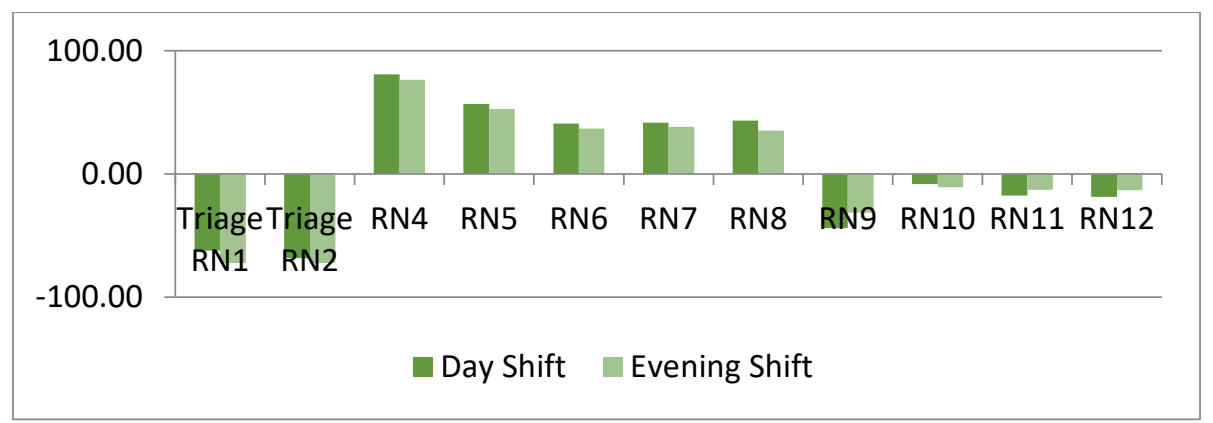

Figure 6. Bottleneck analysis on day and evening shifts nurses

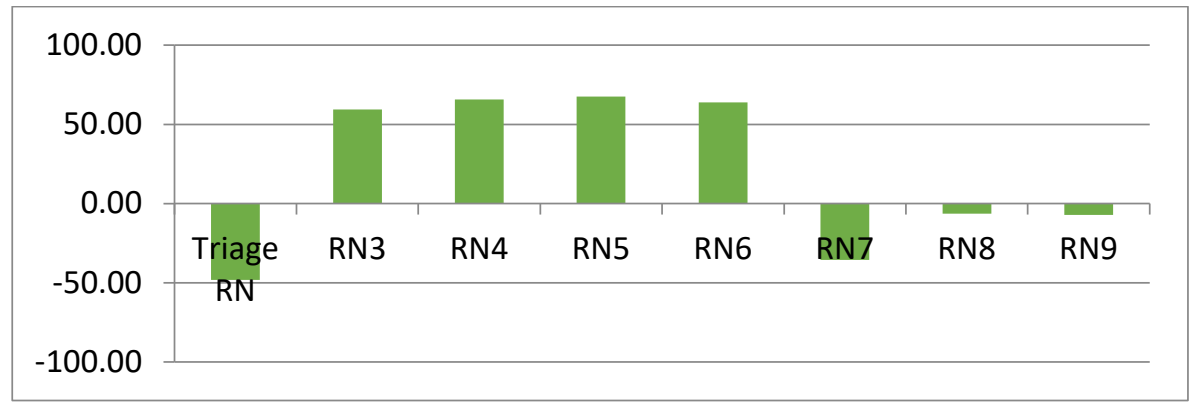

Figure 7. Bottleneck analysis on night shift nurses

\section{Bottleneck reduction}

Based on the feasibility of the ED, following strategies are proposed for the bottleneck reduction: 1) increasing capacity for potential bottleneck stations without changing of the operation time; 2) reducing operation time of potential bottleneck stations without changing of their capacities; and 3) increasing availability of potential limiting resources of physician and nurse.

\subsection{Increasing capacity for potential bottleneck stations without changing of the operation time}

This considers stations of Triage, RU, MTA and ERms with a quantitative comparison of the difference in LOS and WTBS by increasing their capacity. Table 1 shows results of the patient average waiting time and throughput time due to the capacity growth in bottleneck stations. Following solutions are observed.

a) Purely adding triage stations without adding other resources to the current ED system generally has very minor reduction in patient average throughput time, and does not have any improvement in patient average waiting time.

b) Adding two more beds in RU with each nurse serving 7 beds instead of the current 6 does not show much improvement in patient average waiting time and throughput time.

c) The capacity growth in ERms does not see a visible improvement in patient waiting time and throughput time.

d) Increasing capacity in MTA causes the reduction in both patient waiting time and throughput time, and generally leads to a greater reduction in WTBS compared to LOS. Therefore, MTA capacity can be increased for the bottleneck reduction.

\subsection{Reducing operation time of bottleneck stations without changing of their quantities}

This evaluates different processing time scenarios for triage and RU observation so as to determine whether operation time in triage and RU have impact on the patient waiting time to see doctor and their length of stay in the ED, and the level of influence. Tables 2 and 3 display effects of the triage time including registration and RU observation time on average patient waiting time and throughput time respectively. Follows solutions are observed.

a) Operation time reduction in triage has a positive influence on both patient waiting and throughput time, especially with a greater reduction in WTBS compared to LOS.

b) Time reduction in RU observation does reduce the average patient length of stay in the ED, however, reduction in WTBS is rarely observed.

c) Operation time reduction in triage generally leads to a greater reduction in LOS compared to time reduction in RU observation. 
Table 1. Effect of capacity growth in bottleneck stations on patient average waiting and throughput time

\begin{tabular}{llll}
\hline \multirow{2}{*}{ Criteria } & Alternatives & $\begin{array}{l}\text { LOS, Effect (\%) } \\
\text { (Baseline=584 min) }\end{array}$ & $\begin{array}{l}\text { WTBS, Effect (\%) } \\
\text { (Baseline=207 min) }\end{array}$ \\
\hline 1 & Add one Triage1 & -6.68 & 10.63 \\
& Add two Triage1 & -5.48 & -13.04 \\
2 & Add three Triage1 & -1.37 & 5.31 \\
& Add one Triage2 & 9.93 & 62.32 \\
& Add two Triage2 & -4.11 & 70.05 \\
3 & Add three Triage2 & -0.17 & 42.03 \\
4 & Add two beds in RU with each RU nurse & -1.54 & 7.73 \\
& serving 7 beds instead of 6 & & -1.93 \\
& Add one bed in MTA & -4.79 & -27.54 \\
& Add two beds in MTA & -6.34 & -34.78 \\
& Add three beds in MTA & -6.51 & -38.65 \\
& Add four beds in MTA & -5.31 & -22.22 \\
& Add five beds in MTA & -6.16 & 67.63 \\
& Add one bed in Erms & 4.79 & 0.00 \\
\hline
\end{tabular}

* Note: All service stations run 24 hours a day except that Triage2 station is off-shift in the night from 11:30pm to 7:30am.

Table 2. Effect of triage time on average patient waiting time and throughput time

\begin{tabular}{llll}
\hline Criteria & Alternatives & $\begin{array}{l}\text { LOS, Effect (\%) } \\
\text { (Baseline=584 min) }\end{array}$ & $\begin{array}{l}\text { WTBS, Effect (\%) } \\
\text { (Baseline=207 min) }\end{array}$ \\
\hline 1 & $\begin{array}{l}\text { Reduce Triage \& Registration time by 10\% } \\
\text { Reduce Triage \& Registration time by 15\% }\end{array}$ & -14.55 & -47.34 \\
Reduce Triage \& Registration time by 20\% & -23.97 & -28.50 \\
& Reduce Triage \& Registration time by 25\% & -24.14 & -55.56 \\
& $\begin{array}{l}\text { Reduce Triage \& Registration time by 30\% } \\
\text { If Triage \& Registration is complete within [1,2] }\end{array}$ & -24.66 & -34.30 \\
min & & -53.14 \\
If Triage \& Registration is complete within [3,4] & -29.45 & -76.33 \\
min & & -59.90 \\
If Triage \& Registration is complete within [5,6] & -30.31 & -48.79 \\
min & & -60.87 \\
If Triage \& Registration is complete within [7,8] & -25.17 & -57.00 \\
min & & -28.60 \\
If Triage \& Registration is complete within [9,10] \\
min
\end{tabular}

\subsection{Increasing availability of limiting resources in physician and nurse}

Adding additional human resources to the current ED system can be another way to solve the overcrowding issue, but it is only true when the right one is added. Table 4 shows the level of influence on the patient average waiting time and throughput time by adding bottleneck resources in either physician or nurse. Since the hospital is financially available to employ another physician to join the ED team, multiple alternatives are tested to search the best allocation for the physician. Following solutions are observed in the simulation model.

a) Without making changes in the current patient flow, the patient average waiting time and throughput time can be reduced if another physician is allocated to look after patients in ERms and MTA.

b) Adding additional nurse resource in Triage, ERms and MTA does not show an effective improvement of the ED performance. 
Table 3. Effect of RU observation time on average patient waiting time and throughput time

\begin{tabular}{llll}
\hline Criteria & Alternatives & $\begin{array}{l}\text { LOS, Effect (\%) } \\
\text { (Baseline=584 min) }\end{array}$ & $\begin{array}{l}\text { WTBS, Effect (\%) } \\
\text { (Baseline=207 min) }\end{array}$ \\
\hline 1 & Reduce RU time by 10\% & -1.71 & -13.04 \\
& Reduce RU time by 15\% & -5.31 & 65.70 \\
& Reduce RU time by 20\% & -7.02 & 56.04 \\
& Reduce RU time by 25\% & -7.53 & 18.36 \\
& Reduce RU time by 30\% & -10.45 & 10.14 \\
2 & Reduce RU time by 35\% & -10.45 & 17.87 \\
& Release patients in RU 60min in advance & -3.60 & 38.16 \\
& Release patients in RU 90min in advance & -2.91 & 52.66 \\
& Release patients in RU 120min in advance & -4.45 & 79.23 \\
& Release patients in RU 150min in advance & -6.51 & 27.54 \\
& Release patients in RU 180min in advance & -6.68 & 13.53 \\
\hline
\end{tabular}

Table 4. Average waiting and throughput time by adding bottleneck resources in physician and nurse

\begin{tabular}{llll}
\hline Criteria & Alternatives & $\begin{array}{l}\text { LOS, Effect (\%) } \\
\text { (Baseline=584 min) }\end{array}$ & $\begin{array}{l}\text { WTBS, Effect (\%) } \\
\text { (Baseline=207 min) }\end{array}$ \\
\hline 1 & Add one Physician \#5 & -13.53 & -36.71 \\
& Add one Physician \#6 & -8.22 & -35.27 \\
& Add one Physician \#2 & -4.62 & -10.63 \\
& Add one Physician \#3 & -5.31 & -32.37 \\
2 & Add one triage nurse for 24 hrs & 0.17 & 0.00 \\
3 & Add one RN9 \& RN7 (i.e. nurses looking after & -5.31 & 9.18 \\
& patients in ERms \& MTA) & & \\
\hline
\end{tabular}

\subsection{Summary of effective alternatives}

Table 5 summarizes the possible ways that can be used to effectively reduce patient average waiting time to see a doctor and their length of stay in the ED without changes to the current ED patient flow. Their numerical degrees of influence on LOS and WTBS can be refereed back to the previous Tables. With these four summarized alternatives, reducing processing time in triage shows the greatest positive impact on both LOS and WTBS.

Table 5. Effective alternatives to reduce LOS \& WTBS without changes to the current ED patient flow

\begin{tabular}{llll}
\hline \multirow{2}{*}{ Index } & \multirow{2}{*}{ Alternatives } & \multicolumn{2}{l}{ Influence Level } \\
\cline { 3 - 4 } & & LOS & WTBS \\
\hline 1 & Building capacity in MTA & + & ++ \\
2 & Reducing processing time in triage & ++ & ++ \\
3 & Reducing observation time in RU (i.e. Releasing patients in RU faster) & + & - \\
4 & Increasing availability of resource in physician in ERms \& MTA & + & ++ \\
\hline Note: “+” denotes a positive influence; “++” denotes a greater positive influence compared to “+”; “-” denotes a non-effective
\end{tabular}
alternative.

\section{Conclusions}

As the increasing demand for the healthcare service, it is important to improve performance of healthcare systems in the ability to reduce cost, improve care efficiency and quality. A discrete-event simulation (DES) model has been developed in this research to analyze the ED patient flow in a local Hospital. By using the validated model, bottlenecks of the system are identified. The various operating alternatives are tested to relieve bottlenecks in the ED to reduce patients waiting time and length of stay. The validated DES model provides a quantitative tool for the analyst to evaluate the effect level on LOS and WTBS among various operating alternatives in comparison with the baseline measures. Alternative strategies to reduce the detected bottlenecks were effectively examined using the validated simulation model to reduce patient waiting time and throughput time.

This research demonstrates the usefulness of simulation modeling for predicting the impact of various factors to the ED overcrowding. Simulation has shown its power of examining a system to determine its performance and limitation. The simulation model developed in this research can be used to discover various possibilities to improve the service delivery in emergency care systems, such as the current pandemic, COVID-19. 


\section{References}

[1] CIHI. Emergency department visits in 2014 \& 2015: information sheet. Canadian Institute for Health Information. 2015.

[2] Kaushal A, Zhao Y, Peng Q, Strome T, Weldon E, Zhang M, Chochinov A. Evaluation of fast track strategies using agent-based simulation modeling to reduce waiting time in a hospital emergency department. SocioEconomic Planning Sciences. 2015;50:18-31.

[3] Affleck A, Parks P, Drummond A, Rowe BH, Ovens HJ. Emergency department overcrowding and access block. Canadian Journal of Emergency Medicine. 2013;15(6):359-370.

[4] Guttmann A, Schull MJ, Vermeulen MJ, Stukel TA. Association between waiting times and short term mortality and hospital admission after departure from emergency department: population based cohort study from Ontario, Canada. BMJ. 2011;342:d2983.

[5] Carter EJ, Pouch SM, Larson EL. The relationship between emergency department crowding and patient outcomes: a systematic review. Journal of Nursing Scholarship. 2014;46(2):106-115.

[6] Bahari A, Asadi F. A simulation optimization approach for resource allocation in an emergency department healthcare unit. Global Heart. 2020;15(1):14.

[7] Bouzon Nagem Assad D, Spiegel T. Improving emergency department resource planning: a multiple case study. Health Systems. 2020;9(1):2-30.

[8] Weiss SJ, Ernst AA, Nick TG. Comparison of the national emergency department overcrowding scale and the emergency department work index for quantifying emergency department crowding. Academic Emergency Medicine. 2006;13(5):513-518.

[9] Xie Y, Peng Q. Integration of value stream mapping and agent - based modeling for OR improvement. Business Process Management Journal. 2012; 18(4): 585-599.

[10] Higginson I. Emergency department crowding. Emergency Medicine Journal. 2012; 29 (6): 437-443.

[11] Jilani T, Housley G, Figueredo G, Tang PS, Hatton J, Shaw D. Short and Long term predictions of hospital emergency department attendances. International Journal of Medical Informatics. 2019;129:167-174.

[12] Wu X, Xu R, Li J, Khasawneh MT. A simulation study of bed allocation to reduce blocking probability in emergency departments: A case study in China. Journal of the Operational Research Society. 2019;70(8):1376-1390.

[13] Boyle A, Coleman J, Sultan Y, Dhakshinamoorthy V, O'Keeffe J, Raut P, Beniuk K. Initial validation of the International Crowding Measure in Emergency Departments (ICMED) to measure emergency department crowding. Emergency Medicine Journal. 2015;32(2):105-108.

[14] Hoot NR, Aronsky D. Systematic review of emergency department crowding: causes, effects, and solutions. Annals of Emergency Medicine. 2008;52(2):126-136.

[15] Marchesi JF, Hamacher S, Fleck JL. A stochastic programming approach to the physician staffing and scheduling problem. Computers \& Industrial Engineering. 2020;142:106281.

[16] Davis S, Fard N. Theoretical bounds and approximation of the probability mass function of future hospital bed demand. Health Care Management Science. 2020;23(1):20-33.

[17] Bond K, Ospina MB, Blitz S, Afilalo M, Campbell SG, Bullard M, Innes G, Holroyd B, Curry G, Schull M, Rowe BH. Frequency, determinants and impact of overcrowding in emergency departments in Canada: a national survey. Healthcare Quarterly. 2007;10(4):32-40.

[18] Paul SA, Reddy MC, DeFlitch CJ. A systematic review of simulation studies investigating emergency department overcrowding. Simulation. 2010;86(8-9):559-571.

[19] Chalk D. Using computer simulation to model the expansion needs of the ambulatory emergency care unit at Derriford Hospital. Future Healthcare Journal. 2020; 7(1), 60-64.

[20] Zhao Y, Peng Q, Strome T, Weldon E, Zhang M, Chochinov A. Bottleneck detection for improvement of emergency department efficiency. Business Process Management Journal. 2015; 21(3):564-585.

[21] Niu Q, Peng Q, ElMekkawy TY. Improvement in the operating room efficiency using tabu search in simulation. Business Process Management Journal. 2013; 19 (5): 799-818.

[22] Kolker A. Process modeling of emergency department patient flow: Effect of patient length of stay on ED diversion. Journal of Medical Systems. 2008;32(5):389-401.

[23] Mahmoudian-Dehkordi A, Sadat S. A generic simulation model of the relative cost-effectiveness of ICU versus step-down (IMCU) expansion. Journal of Intensive Care Medicine. 2020;35(2):191-202.

[24] Jurishica CJ. Emergency department simulations: medicine for building effective models. In: Proceedings of the Winter Simulation Conference. 2005.

[25] Ordu M, Demir E, Tofallis C, Gunal MM. A novel healthcare resource allocation decision support tool: A forecasting-simulation-optimization approach. Journal of the Operational Research Society. 2020:1-6. 
[26] Saghafian S, Austin G, Traub SJ. Operations research/management contributions to emergency department patient flow optimization: Review and research prospects. IIE Transactions on Healthcare Systems Engineering. 2015;5(2):101-123.

[27] McAvoy S, Staib A, Treston G. Can a system dynamics model of the emergency department show which levers reduce bottlenecks and delays to improve access to care?. Systems Research and Behavioral Science. 2020:1-19.

[28] Günal MM, Pidd M. Discrete event simulation for performance modelling in health care: a review of the literature. Journal of Simulation. 2010;4(1):42-51.

[29] Leporis M, Králová Z. A simulation approach to production line bottleneck analysis. In: International Conference Cybernetics and Informatics. 2010. p. 13-22.

(C) 2020 by the author(s). This work is licensed under a Creative Commons Attribution 4.0 International License (http://creativecommons.org/licenses/by/4.0/). Authors retain copyright of their work, with first publication rights granted to Tech Reviews Ltd. 